International Journal of Pure and Applied Mathematics

Volume 87 No. $6 \quad 2013,809-815$

ISSN: 1311-8080 (printed version); ISSN: 1314-3395 (on-line version)

url: http://www.ijpam.eu

doi: http://dx.doi.org/10.12732/ijpam.v87i6.9

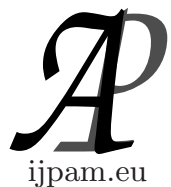

\title{
ON STRONG IFP NEAR-RINGS
}

\author{
P. Dheena ${ }^{1}$, B. Elavarasan ${ }^{2}$ \\ ${ }^{1}$ Department of Mathematics \\ Annamalai University \\ Annamalainagar, 608 002, INDIA \\ ${ }^{2}$ Department of Mathematics \\ Karunya University \\ Coimbatore, 641 114, Tamilnadu, INDIA
}

\begin{abstract}
In this paper, we introduce the notion of strong IFP and weak IFP near-rings. Weak IFP near-ring is a generalization of IFP near-ring. We study the basic properties of right weak IFP near-rings. We show that if $N$ is a 2-primal near-ring and if $N$ is strong IFP, then $N$ is left weakly regular if and only if every prime ideal of $N$ is maximal.
\end{abstract}

AMS Subject Classification: $16 Y 30$

Key Words: regular, IFP near-ring, reduced, 2-primal and weakly regular

\section{Introduction}

Throughout this paper, $N$ denotes a zero-symmetric near-ring not necessarily with identity unless otherwise stated. Let $P(N)$ denote the prime radical and $N(N)$ the set of nilpotent elements of the near-ring $N$. For $X \subseteq N, l(X)$ (resp. $r(X))$ and $\langle x\rangle$ denote the left (resp. right) annihilator of $X$ and the ideal of $N$ generated by $x$ respectively.

For any subsets $A, B$ of $N$, we denote $(A: B)=\{n \in N / n B \subseteq A\}$. It is trival to check that if $A$ is left ideal of $N$ and $B$ is a $N$-subgroup of $N$, then $(A: B)$ is an ideal of $N$ by [8, Corollary 1.43].

Received: September 6, 2013

(C) 2013 Academic Publications, Ltd. url: www.acadpubl.eu 
A near-ring $N$ is said to be reduced if $N(N)=0$. A near-ring $N$ is said to be regular if for any $a \in N$, there exists $x \in N$ such that $a=a x a$.

Recall that a near-ring $N$ is said to be 2-primal if $P(N)=N(N)$. A nearring $N$ is subdirectly irreducible if $N$ has nonzero intersection of nonzero ideals. A near-ring $N$ is said to be strong IFP if $x y \in P(N)$ implies $x N y=0$ for $x, y \in N$. A near-ring $N$ is said to be IFP if $a b=0$ implies $a n b=0$ for all $n \in N$ and $a, b \in N$. Clearly every strong IFP near-ring is a IFP near-ring. If $N$ is reduced, then the notions of IFP and strong IFP coincide

A near-ring $N$ is said to be left weak IFP if $a b=0$ for $a(\neq 0), b \in N$ implies $a^{\prime} N b=0$ for some $\left.a^{\prime}(\neq 0) \in<a\right\rangle$. The right weak IFP can be defined symmetrically. A near-ring $N$ is said to be weak IFP if $a b=0$ for any nonzero elements $a, b \in N$ implies $a^{\prime} N b^{\prime}=0$ for some $a^{\prime}(\neq 0) \in<a>$ and $b^{\prime}(\neq 0) \in<b>$.

Clearly IFP near-ring is a weak IFP near-ring, but the converse need not be true as the following example shows.

Example 1.1. Let $N=\left(\begin{array}{cc}F & F \\ 0 & F\end{array}\right)$ where $F=\{0,1\}$ is the field under addition and multiplication modulo 2. Then $N$ is a weak IFP near-ring but not IFP near-ring, since if $x=\left(\begin{array}{ll}1 & 0 \\ 0 & 0\end{array}\right)$ and $y=\left(\begin{array}{ll}0 & 0 \\ 0 & 1\end{array}\right)$, then $x y=0$ and $x N y \neq 0$. Here $N$ is neither left weak IFP nor right weak IFP.

Clearly every strong IFP near-rings are IFP near-rings, however IFP nearring need not be strong IFP as can be seen by the following example.

Example 1.2. Let $(N,+)$ (where $N=\{0, a, b, c\}$ ) be the klein's four group. Define multiplication in $N$ as follows

\begin{tabular}{c|cccc}
$\cdot$ & 0 & $\mathrm{a}$ & $\mathrm{b}$ & $\mathrm{c}$ \\
\hline 0 & 0 & 0 & 0 & 0 \\
$\mathrm{a}$ & 0 & $\mathrm{a}$ & $\mathrm{b}$ & $\mathrm{c}$ \\
$\mathrm{b}$ & 0 & 0 & 0 & 0 \\
$\mathrm{c}$ & 0 & $\mathrm{a}$ & $\mathrm{b}$ & $\mathrm{a}$
\end{tabular}

Then $(N,+,$.$) is a near-ring (see Pilz[8], P-408, Scheme-11) which is a IFP$ near-ring but not a strong IFP near-ring, since $a b \in P(N)$, but $a N b \neq 0$.

Clearly every reduced near-ring is a 2-primal and strong IFP near-ring, but the converse need not be true as the following example shows.

Example 1.3. Let $(N,+)$ (where $N=\{0, a, b, c\}$ ) be the klein's four group. Define multiplication in $N$ as follows 


\begin{tabular}{c|cccc}
$\cdot$ & 0 & $\mathrm{a}$ & $\mathrm{b}$ & $\mathrm{c}$ \\
\hline 0 & 0 & 0 & 0 & 0 \\
$\mathrm{a}$ & 0 & $\mathrm{a}$ & 0 & $\mathrm{a}$ \\
$\mathrm{b}$ & 0 & 0 & 0 & 0 \\
$\mathrm{c}$ & 0 & $\mathrm{a}$ & 0 & $\mathrm{a}$
\end{tabular}

Then $(N,+,$.$) is a near-ring (see Pilz[8], P-408, Scheme-12) which is a 2-$ primal and strong IFP near-ring, but not reduced.

G.F.Birkenmeier, J.Y.Kim and J.K.Park [2] have shown that a reduced ring $R$ is weakly regular if and only if every prime ideal of $R$ is maximal. We extend this result to strong IFP near-rings which are 2-primal. For basic terminology in near-ring we refer to Pilz [8].

\section{Main Results}

Lemma 2.1. Let $N$ be a near-ring with identity. If $N$ is left weak IFP, then for any $x, y \in N$ with $x y=1$ implies $y x=1$.

Proof. Let $N$ be a left weak IFP near-ring and $x y=1$. Suppose $y x \neq 1$. Then $(1-y x) y x=0$. Since $N$ is left weak IFP, we have $x^{\prime} N y x=0$ for some $x^{\prime}(\neq 0) \in<1-y x>$. Now, $x^{\prime} N y=x^{\prime} N y x y=0$. Then $x^{\prime}=x^{\prime} x y \in x^{\prime} N y=0$, a contradiction.

Proposition 2.2. Let $N$ be a regular near-ring. Then the following conditions are equivalent:

i) $N$ is a right weak IFP near-ring

ii) If $x(\neq 0) \in r(a)$, then $r(a)$ contains a non-zero ideal $I$ with $I \subseteq\langle x\rangle$

iii) If $x(\neq 0) \in r(a), r(a N)$ contains a non-zero ideal $I$ with $I \subseteq\langle x\rangle$

iv) If $x(\neq 0) \in r(a), i \in r(a N)$ for some $i(\neq 0) \in<x>$

Proof. $i) \Rightarrow$ ii) Let $x(\neq 0) \in r(a)$. Then $a N x^{\prime}=0$ for some non-zero element $x^{\prime} \in\langle x\rangle$. For any $n \in N, x^{\prime} n a=\left(x^{\prime} n a\right) t\left(x^{\prime} n a\right)=x^{\prime} n\left(a t x^{\prime}\right) n a=0$ for some $t \in N$. Thus $x^{\prime} N a=0$ and so $\left\langle x^{\prime}>N a=0\right.$. Let $y \in\left\langle x^{\prime}\right\rangle$. Then by regularity of $N$, we have $a y=0$. Thus $\left.a<x^{\prime}\right\rangle=0$.

ii) $\Rightarrow$ iii) Let $x(\neq 0) \in r(a)$. Then $a I=0$ and so $a N I=0$.

iii) $\Rightarrow i v$ ) It is obvious.

$i v) \Rightarrow i)$ Let $a b=0$ for $a(\neq 0), b \in N$. Then $b^{\prime} \in r(a N)$ for some $b^{\prime}(\neq 0) \in<$ $b>$. Thus $a N b^{\prime}=0$.

We now give an example to show that Proposition 2.2 is not true if $N$ is not a regular near-ring. 
Example 2.3. Consider the dihedral group $N=\{0, a, 2 a, 3 a, b, a+b, 2 a+$ $b, 3 a+b\}$ with addition and multiplication defined as in Pilz ([9, P-339, Scheme$2])$.

\begin{tabular}{c|cccccccc}
$\cdot$ & 0 & $\mathrm{a}$ & $2 \mathrm{a}$ & $3 \mathrm{a}$ & $\mathrm{b}$ & $\mathrm{a}+\mathrm{b}$ & $2 \mathrm{a}+\mathrm{b}$ & $3 \mathrm{a}+\mathrm{b}$ \\
\hline 0 & 0 & 0 & 0 & 0 & 0 & 0 & 0 & 0 \\
$\mathrm{a}$ & 0 & $\mathrm{a}$ & $2 \mathrm{a}$ & $3 \mathrm{a}$ & $\mathrm{b}$ & $\mathrm{a}+\mathrm{b}$ & $2 \mathrm{a}+\mathrm{b}$ & $3 \mathrm{a}+\mathrm{b}$ \\
$2 \mathrm{a}$ & 0 & $2 \mathrm{a}$ & 0 & $2 \mathrm{a}$ & 0 & 0 & 0 & 0 \\
$3 \mathrm{a}$ & 0 & $3 \mathrm{a}$ & $2 \mathrm{a}$ & $\mathrm{a}$ & $\mathrm{b}$ & $\mathrm{a}+\mathrm{b}$ & $2 \mathrm{a}+\mathrm{b}$ & $3 \mathrm{a}+\mathrm{b}$ \\
$\mathrm{b}$ & 0 & $\mathrm{~b}$ & $2 \mathrm{a}$ & $\mathrm{b}$ & $\mathrm{b}$ & 0 & $2 \mathrm{a}+\mathrm{b}$ & 0 \\
$\mathrm{a}+\mathrm{b}$ & 0 & $\mathrm{a}+\mathrm{b}$ & 0 & $3 \mathrm{a}+\mathrm{b}$ & 0 & $\mathrm{a}+\mathrm{b}$ & 0 & $3 \mathrm{a}+\mathrm{b}$ \\
$2 \mathrm{a}+\mathrm{b}$ & 0 & $2 \mathrm{a}+\mathrm{b}$ & $2 \mathrm{a}$ & $2 \mathrm{a}+\mathrm{b}$ & $\mathrm{b}$ & 0 & $2 \mathrm{a}+\mathrm{b}$ & 0 \\
$3 \mathrm{a}+\mathrm{b}$ & 0 & $3 \mathrm{a}+\mathrm{b}$ & 0 & $\mathrm{a}+\mathrm{b}$ & 0 & $\mathrm{a}+\mathrm{b}$ & 0 & $3 \mathrm{a}+\mathrm{b}$
\end{tabular}

Then $(N,+,$.$) is a near-ring. Clearly (N,+,$.$) is a right weak IFP and$ $a+b \in r(b)$, but $r(b)$ does not contains a non-zero ideal $I$ with $I \subseteq<a+b\rangle$.

Proposition 2.4. Let $N$ be a near-ring with identity. Then the following conditions are equivalent:

i) $N$ is a left weak IFP near-ring

ii) If $x(\neq 0) \in l(a)$, then $l(a)$ contains a non-zero ideal $I$ with $I \subseteq<x>$

iii) If $x(\neq 0) \in l(a)$, then $l(N a)$ contains a non-zero ideal $I$ with $I \subseteq<x>$

iv) If $x(\neq 0) \in l(a)$, then $i \in l(N a)$ for some $i(\neq 0) \in<x>$

Proof. Proof is as similar in Proposition 2.2.

Lemma 2.5. Let $N$ be a regular near-ring. If $N$ is subdirectly irreducible, then the following conditions are equivalent:

i) $N$ is a right weak IFP near-ring

ii) if $x(\neq 0) \in r(S)$, then $r(S)$ contains a non-zero ideal $I$ of $N$ with $I \subseteq<x>$ for any subset $S$ of $N$.

Proof. $i) \Rightarrow i i)$ Let $N$ be a right weak IFP near-ring and $x \in r(S)$ for any subset $S$ of $N$. For any $s_{i} \in S$, by Proposition 2.2, we have $r\left(s_{i}\right)$ contains a non-zero ideal $I_{i}$ of $N$ with $I_{i} \subseteq<x>$ and so $0 \neq \cap I_{i} \subseteq<x>$ with $S\left(\cap I_{i}\right)=0$.

$i i) \Rightarrow i$ ) It is trivial.

Proposition 2.6. Let $N$ be a regular near-ring. If $N$ is subdirectly irreducible, then the following conditions are equivalent:

i) $N$ is a right weak IFP near-ring

ii) $N$ is a reduced near-ring

iii) $N$ is a strong IFP near-ring

iv) $N$ is a IFP near-ring. 
Proof. $i) \Rightarrow i i)$ Let $a(\neq 0) \in N$ such that $a^{2}=0$. Since $N$ is regular, we have $a=a x a$ for some $x \in N$. Set $e=a x$. Let $S=\{n-n e / n \in N\}$. Then $r(S)$ contains a non-zero ideal $J$ with $J \subseteq<e>$ and so $n j=n e j$ for all $n \in N$ and for all $j \in J$. Let $j(\neq 0) \in J$. Then there exists $y \in N$ such that $j=j y j=j(y j)=j e(y j)=j a(x y j)=(j a) e(x y j)=j a^{2} x^{2} y j=0, \mathrm{a}$ contradiction.

ii) $\Rightarrow$ iii) It follows from Proposition 2.94 of [8].

$i i i) \Rightarrow i v)$ and $i v) \Rightarrow i$ ) are trival.

Hereafter $N$ denote a zero-symmetrc near-ring with left identity. Following G. F. Birkenmeier and N. J. Groenewald [1], a near-ring $N$ is said to be left (resp. right) weakly $\pi$-regular if $x^{n} \in<x^{n}>x^{n}$ (resp. $x^{n} \in x^{n}<x^{n}>$ ) for all $x \in N$ and for some natural number $n=n(x)$. A near-ring $N$ is called weakly $\pi$-regular if $N$ is both left and right weakly $\pi$-regular. A weakly $\pi$-regular near-ring is called weakly regular when $n=1$.

A near-ring $N$ is said to be left (resp. right) pseudo $\pi$-regular if $x^{n} \in<$ $x>x^{n}$ (resp. $x^{n} \in x^{n}<x>$ ) for all $x \in N$ and for some natural number $n=n(x)$. A near-ring $N$ is called pseudo $\pi$-regular if $N$ is both left and right pseudo $\pi$-regular.

Proposition 2.7. Let $P$ be a completely prime ideal of $N$. If $N / P(N)$ is a left weakly $\pi$-regular near-ring, then $P$ is a maximal ideal of $N$.

Proof. Let $P$ be a completely prime ideal of $N$ and $N / P(N)$ be a left weakly $\pi$-regular near-ring. Suppose $M$ is an ideal of $N$ such that $P \subset M$. Let $a \in M \backslash P$. Then $P+<a>\subseteq M$. Since $N / P(N)=\bar{N}$ is a left weakly $\pi$-regular, we have $\bar{N} \bar{a}^{n}=<\bar{a}^{n}>\bar{a}^{n}$ for some positive integer $n$. So $\bar{N} \bar{a}^{n}=\bar{M} \bar{a}^{n}$. Hence $\bar{a}^{n}=\bar{b} \bar{a}^{n}$ for some $\bar{b} \in \bar{M}$ and so $(1-b) a^{n} \in P$ which implies $N=M$.

Corollary 2.8. (5 Theorem 2.3) Let $P$ be a completely prime ideal of a ring $R$. If $P / P(R)$ is a left weakly $\pi$-regular near-ring, then $P$ is a maximal ideal of $R$.

G.F.Birkenmeier, J.Y.Kim and J.K.Park [2] have shown that a reduced ring $R$ is weakly regular if and only if $R$ is right weakly regular and if and only if every prime ideal of $R$ is maximal. We shall prove this result under generalized conditions.

Proposition 2.9. Let $N$ be a 2-primal near-ring. If $N$ is strong IFP, then the following conditions are equivalent:

i) $N$ is left weakly regular

ii) $N$ is left weakly $\pi$-regular

iii) $N / P(N)$ is left weakly $\pi$-regular 
iv) $N / P(N)$ is left pseudo $\pi$-regular

v) Every prime ideal of $N$ is maximal.

Proof. $i) \Rightarrow i i), i i) \Rightarrow i i i)$ and $i i i) \Rightarrow i v)$ Proofs are trival.

$i v) \Rightarrow v)$ It follows from Corollary 3.10 of [1].

$v) \Rightarrow i$ ) Suppose $N$ is not a left weakly regular. Then there exists an element $a \in N$ such that $a \notin\langle a>a$. Let $T$ be a union of all prime ideals of $N$, such that each of them contain $a$. Let $S=N \backslash T$. Then $S$ is a multiplicative closed subset of $N$ by Theorem 5 of [4]. Let $F$ be the multiplicative closed system generated by $\{a\} \cup S$. Suppose $0 \notin F$. Then there exists a proper prime ideal $M$ of $N$ with $M \cap F=\phi$ by Proposition 2.81 of [8]. Since $a \notin M$, we have $M+\langle a\rangle=N$ and so there exists $b \in M$ and $c \in\langle a\rangle$ such that $b+c=1$. Clearly $b \notin T$, which implies $b \in F \cap M=\phi$, a contradiction. Thus $0 \in F$.

So $0=a^{n_{1}} s_{1} a^{n_{2}} \ldots a^{n_{t}} s_{t}$ where $s_{i} \in S$ and $n_{1}, n_{2}, \ldots, n_{t}$ are positive integers. For any prime ideal $P$, we have $a^{n_{1}} s_{1} a^{n_{2}} \ldots a^{n_{t}} s_{t} \in P$. Since $P$ is completely prime, we have $a \in P$ or $s_{i} \in P$ for some $i$. Let $s=s_{1} s_{2} \ldots s_{t}$. Then for any prime ideal $P$; either $a \in P$ or $s \in P$. Then $s a \in P(N)$. Since $N$ is a strong IFP nearring, we have $s N a=0$. Then $\langle s\rangle N a=0$. Observe that a prime ideal can not contains both $a$ and $s$; otherwise a prime ideal would contain both of them, which contradicts the definitions of $S$ and $T$ which implies $\langle s\rangle+\langle a\rangle=N$ and so $N$ is a left weakly regular near-ring.

Corollary 2.10 (2, Theorem 8). Let $R$ be a reduced ring. Then the following conditions are equivalent:

i) $R$ is weakly regular

ii) $R$ is right weakly $\pi$-regular

iii) Every prime ideal of $R$ is maximal

Proof. The proof is an immediate consequence of Proposition 2.9 and Theorem 12 of [3].

Corollary 2.11 (2, Corollary 9). Let $R$ be a 2-primal ring. Then the following conditions are equivalent:

i) $R / P(R)$ is weakly regular

ii) $R / P(R)$ is right weakly regular

iii) Every prime ideal of $R$ is maximal.

\section{References}

[1] G. F. Birkenmeier and N. J. Groenewald, Near-ring in which each prime factor is simple, Mathematics Pannonica, 10, 257 - 269 (1999). 
[2] G.F. Birkenmeier, J.K.Kim and J.K.Park, A connection between weak regularity and the simplicity of prime factor rings, Proc. Amer. Math. Soc. 122, 53 - 58 (1991).

[3] P. Dheena and D. Sivakumar, Left prime weakly regular near-rings, Tamkang J. Math., 36(4), 309 - 313 (2005).

[4] P. Dheena and G. Satheeskumar, Completely 2-primal ideals in 2-primal near-rings, Tamsui Oxf. J. Math. Sci., to be appear.

[5] C.Y.Hong, N.K.Kim and T.K.Kwak, On rings whose prime ideals are maximal, Bull. Korean. Math. Soc. 37, 1 - 9 (2000).

[6] C.Y.Hong, Y.C.Jeon, K.H.Kim, N.K.Kim and Y.Lee, Weakly regular rings with $A C C$ on annihilators and maximality of strongly prime ideals of weakly regular rings, J. Pure Appl. Math, 207, 565 - 574 (2006).

[7] S. U. Hwang, Y. C. Jeon and K. S. Park, A generalization of insertion-offactors-property, Bull. Korean Math. Soc., 44, 87 - 94 (2007).

[8] G. Pilz, Near-Rings, North-Holland, Amsterdam, 1983. 
